\title{
Editorial: Nanoscience and Nanomaterials for the Knowledge and Conservation of Cultural Heritage
}

\author{
Luca Tortora $^{1,2 \star}$, Gabriella Di Carlo ${ }^{3}$, Maria Jesus Mosquera ${ }^{4}$ and Gabriel Maria Ingo ${ }^{3}$ \\ ${ }^{1}$ LASR3 Surface Analysis Laboratory, National Institute for Nuclear Physics (INFN) Roma Tre Division, Rome, Italy, \\ ${ }^{2}$ Department of Sciences, Roma Tre University, Rome, Italy, ${ }^{3}$ Institute for the Study of Nanostructured Materials (ISMN), \\ National Research Council (CNR), Rome, Italy, ${ }^{4}$ Departamento de Quimica Fisica, Facultad de Ciencias, Universidad de \\ Cadiz, Cadiz, Spain
}

Keywords: conservation, protection, cleaning, diagnostics, consolidants, corrosion, coatings, paintings

\section{Editorial on the Research Topic}

Nanoscience and Nanomaterials for the Knowledge and Conservation of Cultural Heritage

OPEN ACCESS

Edited by:

Saulius Juodkazis, Swinburne University of Technology, Australia

Reviewed by: Soon Hock Ng, Swinburne University of Technology, Australia

*Correspondence: Luca Tortora Iuca.tortora@uniroma3.it

Specialty section: This article was submitted to Colloidal Materials and Interfaces, a section of the journal

Frontiers in Materials

Received: 14 September 2020 Accepted: 06 October 2020 Published: 29 October 2020

Citation:

Tortora L, Di Carlo G, Mosquera MJ and Ingo GM (2020) Editorial: Nanoscience and Nanomaterials for the Knowledge and Conservation of

Cultural Heritage.

Front. Mater. 7:606076. doi: 10.3389/fmats.2020.606076
"To protect and to serve" is the motto of the Los Angeles Police Department, adopted also by other law enforcement agencies; it underlines the necessity of protecting citizens from criminal acts and at the same time serving the community. Well, considering that works of art constitute a priceless good and they should be protected as such, this motto could be associated even to scientists working in the cultural heritage field. In fact, it is possible to safeguard the cultural heritage working for example on the development of innovative materials as protective agents or explaining degradation mechanisms occurring in artworks by using innovative materials and methods like those based on the nanotechnologies science.

In the last decade, emerging organic and inorganic nanomaterials have been designed, synthesized, and characterized for the really non-invasive and selective cleaning, the reliable consolidation, and the long-lasting reliable conservation also under unsuitable climate conditions. At the same time, sophisticated analytical techniques are more frequently combined to understand the nano and micro-chemical and structural features of artworks for the comprehensive knowledge of their "material" nature and the deciphering of manufacturing processes.

The structure of this special issue reflects these two aspects. It is composed of six original research articles dealing with the application of innovative nanomaterials for the protection of different substrates (archeological wood, steel, and stone material) and cleaning techniques for the disinfection of historical leather and selective removal of polymeric material. In addition, this special topic collection presents seven research articles describing the application of combined advanced analytical techniques to unveil different degradation mechanisms.

For what concerns the nanomaterials for the conservation of cultural heritage, Antonelli et al. report an article where they tested three different nanoscale consolidants to protect waterlogged archaeological wood samples. Lignin nanoparticles, bacterial nanocellulose, and cellulose nanocrystals were used on wood samples coming from two Italian archaeological sites. Problems related to color changing and differences in penetration of the nano-consolidants were extensively discussed. The paper proposed by Giuliani et al. shows a new generation of smart materials able to release the loaded inhibitor depending on the chemical environmental 
conditions. Three inhibitors containing a carboxylic moiety, such as p-aminobenzoic, succinic, and caffeic acids were studied as protective agents of corrosion in simulated concrete pore solutions containing chloride ions; p-aminobenzoic acid was then used for the functionalization of silica nanoparticles and LDHs, adopted as nanocarriers for the inhibitors.

Seems to be a great deal of interest for the consolidant products used for stone. In fact, Ruggiero et al. propose a coating composed of a tetraethoxyorthosilicate matrix (TEOS), an elasticiser [hydroxyl-terminated polydimethylsiloxane (PDMS-OH)] and a non-ionic surfactant (n-octylamine). This TEOS-based composite coating was then functionalized with two different silica nanofillers with antifouling properties: commercial $\mathrm{TiO}_{2}$ nanoparticles and two different silica nanocontainers, loaded with the commercial biocide 2mercaptobenzothiazole. In the same collection, Kapetanaki et al. reported a procedure for eco-friendly and one-pot synthesis of a consolidant incorporating in TEOS, PDMS, and nanoparticles of synthesized calcium oxalate. Cement mortars treated with this colloidal solution exhibited wellpreserved aesthetic surface parameters and significant maintenance of the treatment.

Particular attention was also payed paid to cleaning methods. X-ray beams were used by Vadrucci et al. for the sterilization of leather wallpaper fragments dating back to the 1700s. The disinfection treatment permitted to eliminate the biofilms present on the leather and produced by the following microorganisms: B. cereus and M. timonae. On the other hand, a chemical approach for the removal of aged or detrimental polymeric coatings from artworks was investigated by Baglioni et al. The interaction between nanostructured fluids (NSFs), usually adopted by restorers for cleaning procedures, and four different kinds of acrylic and vinyl polymer films deposited onto glass, marble, and polystyrene substrates was studied.

Integrated methodological approaches based on the combined use of complementary surface, interfaces, and bulk analytical techniques were applied on different heritage objects and materials as like inks, gilded copperbased buckles, historical metal plates, gilded diadem fragments, gilded plaques, and ancient brass wind instruments. Saviello et al. analyzed blue and black writing inks. Results coming from Raman spectroscopy and surface enhanced Raman scattering (SERS) revealed that all analyzed writing inks are composed of Crystal Violet and other triarylmethane dye mixtures, with, in some cases, the addition of phthalocyanine dyes. Raman and SERS were used even to investigate related aging processes due to the presence of light and high temperatures. Degradation processes were also studied by Graziani et al. In this case, time of flight secondary ion mass spectrometry (ToF-SIMS) depth profiling experiments and SEM-energy dispersive X-ray spectroscopy (EDS) were combined to spatially locate corrosion products present in a fire gilded brooch dating back to 16th-17th century $\mathrm{AD}$ and found during the dredging of the Tiber river (Rome, Italy). On the contrary, based on a non-destructive alternative, Lins et al. proposed an analytical approach to determine the gilding thickness for a similar gilded copper-based buckle. An innovative algorithm was developed to measure thin metallic layers deposited on a sample surface taking advantage of differential X-ray attenuation in MA-XRF analysis. Even in the article of Albini et al., the protagonists are copper-based objects. Painted metal surfaces were fully characterized by a plethora of complementary spectroscopic techniques such as Fourier transform infrared spectroscopy (FTIR), energydispersive X-Ray fluorescence spectroscopy (EDXRF), high spatial resolution field emission scanning electron microscopy coupled with energy dispersive X-ray spectroscopy (EDS), and optical microscopy (OM). In particular, FTIR analysis revealed degradation products generated from the interaction between the organic binding media and metal substrates. A detailed study aimed to understand the manufacturing techniques used to give the gold or silver appearance in metal artifacts from ancient Peru was afforded by Ingo et al.. In the article, different metal objects, some of them dating back around 300-400 AD, found in the tombs of the Lords of Sipán (Lambayeque, Peru) and of the Lady of Cao (El Brujo, Peru) were characterized using complementary techniques as $\mathrm{OM}$, SEM-EDS, X-ray diffraction (XRD), and X-ray photoelectron spectroscopy (XPS). The abovementioned combined analytical approach permitted to the authors to have a detailed picture of the degradation process resulting from interactions between soil species and $\mathrm{Ag}-\mathrm{Cu}-\mathrm{Au}$ ternary alloys.

What happens when the surface of a brass comes in contact with saliva? Can be the saliva considered a potential corrosion agent? The problem was addressed by Fantauzzi et al. In the article, artificial saliva was placed in contact with brass surfaces, and the composition and morphology of the film formed after the chemical interaction were was investigated by XPS, SEM, and electrochemical impedance spectroscopy (EIS). Finally, the paper from Grottoli et al. shows the potentialities of the metagenomics applied to the cultural heritage conservation. A micro-invasive sampling method that involves the use of a simple adhesive tape followed by DNA extraction and PCR amplification permitted to identify the microbial communities colonizing the surface of the hypogeum of the Basilica di San Nicola in Carcere Church in Rome.

In conclusion, as you will appreciate, this special issue contains several hints and suggestions that fall in different fields of application strictly related to the cultural heritage. We hope that it can be a useful tool for conservation scientists, restorers, curators, or simply art lovers. 


\section{AUTHOR CONTRIBUTIONS}

All authors contributed equally to this article and have made a substantial, direct and intellectual contribution to the work, and approved it for publication.

\section{ACKNOWLEDGMENTS}

Topic Editors would like to thank all the authors for their contribution to this Special Issue. And, a great thank you also goes to reviewers and editorial staff from Frontiers in Materials and Frontiers in Chemistry for the successful completion of this special collection.

Conflict of Interest: The authors declare that the research was conducted in the absence of any commercial or financial relationships that could be construed as a potential conflict of interest.

Copyright (C) 2020 Tortora, Di Carlo, Mosquera and Ingo. This is an open-access article distributed under the terms of the Creative Commons Attribution License (CC $B Y)$. The use, distribution or reproduction in other forums is permitted, provided the original author(s) and the copyright owner(s) are credited and that the original publication in this journal is cited, in accordance with accepted academic practice. No use, distribution or reproduction is permitted which does not comply with these terms. 